\title{
Manifestaciones respiratorias de las enfermedades reumáticas en niños
}

\author{
Dr. Vicente D’Arago', Dra. Bebella Nieto', Dra. Crhisol Meléndez² \\ 1 Neumonólogos Pediatras, Médicos Especialistas de la Clínica Razetti de Barquisimeto, Barquisimeto, Estado Lara, Venezuela. \\ 2 Neumonólogo Pediatra, Médico Especialista del Centro Médico San Francisco, Barquisimeto, Estado Lara, Venezuela.
}

\section{RESPIRATORY MANIFESTATION OF RHEUMATIC DISEASE IN CHILDREN}

Rheumatic diseases are a heterogeneous group of autoimmune basis and mainly joint and skin manifestations. Respiratory manifestations of these are less common in children than in adults, can affect all respiratory structures, however the most common are the pleural space with pleuritis and effusion and interstitial lung disease. Alterations are usually subclinical beginning, which are evident to the tomography of chest or lung function tests as spirometry or diffusion test for carbon monoxide. Some cases presents with severe respiratory impairment as respiratory bleeding or pneumonia. The comprehensive evaluation included clinical history, imaging studies and pulmonary function is key in the evaluation and management of these patients.

Key words: Rheumatic diseases, respiratory manifestations, children.

\section{RESUMEN}

Las enfermedades reumáticas son un grupo heterogéneo de patologías de base autoinmune y manifestaciones principalmente articulares y cutáneas. Las expresiones respiratorias de estas en niños son menos frecuentes que en los adultos, pueden afectar todas las estructuras respiratorias, sin embargo las más frecuentes son el espacio pleural con pleuritis y derrame y la afectación intersticial pulmonar. Las alteraciones suelen ser de comienzo subclínico, que se hacen evidentes a la exploración con tomografía de tórax o en pruebas de función respiratoria como espirometría o prueba de difusión de monóxido de carbono. Algunos casos cursan con afectación respiratoria severa, como hemorragia respiratoria o neumonías sobre agregadas. La evaluación integral con historia clínica, estudios de imágenes y de función respiratoria son la clave en la evaluación y manejo de estos pacientes. Palabras clave: Enfermedades reumáticas, manifestaciones respiratorias, niños.

\section{INTRODUCCIÓN}

Las enfermedades reumáticas constituyen un grupo heterogéneo de enfermedades de curso habitualmente crónico, que afectan al tejido conectivo, principal componente del sistema musculo esquelético y además de múltiples órganos del cuerpo entre los que se encuentra el pulmón. En los niños como en los adultos tienen diversas formas de presentación y pronóstico, que va desde cuadro auto limitados hasta potencialmente fatales.

La afectación pulmonar en pacientes con enfermedades reumatológicas puede comprometer cualquier zona del aparato respiratorio: vía aérea, vasos sanguíneos, parénquima, pleura y músculos respiratorios ${ }^{(1,2)}$ (Figura I). Es de suma importancia conocer las manifestaciones respiratorias de estas patologías en el niño, porque su presentación en este grupo

Correspondencia: Dr. Vicente D'Arago

Neumonólogo Pediatra, Médico Especialista de la Clínica Razetti de Barquisimeto, calle 27 con carrera 21, anexo torre cristal, 2do. Piso, consultorio 216, Barquisimeto, Estado Lara, Venezuela. Código Postal 3001.

Teléfono: +582512329459, +584145249743.

E-mail:vdaragos@hotmail.com

ISSN 07 |8-332| Derechos reservados. de edad pueden estar relacionada con una alta morbilidad, mortalidad y en muchos casos puede llegar a ser la presentación clínica inicial predominante(2). Otro aspecto relevante, es que su forma de presentación pulmonar puede ser similar a otras patologías, por lo que su diagnostico diferencial es amplio pudiendo confundirse con enfermedades infecciosas, oncohematológicas, inmunodeficiencias entre otras ${ }^{(2)}$.

La frecuencia, la severidad y el tipo de afección pulmonar varían en función de la enfermedad prevalente. Los mecanismos del daño pulmonar en las enfermedades reumática no son totalmente claros, sin embargo, debido a su vascularización el pulmón constituye un objetivo inmunológico para estas enfermedades ${ }^{(2,3)}$. Las manifestaciones respiratorias de estas enfermedades pueden ser: I) Por daño directo de la enfermedad de base; 2) Secundaria a infecciones; 3) Secundaria a los efectos tóxicos de las drogas involucradas en el tratamiento ${ }^{(3)}$.

Por otra parte, estas manifestaciones respiratorias pueden ser agudas potencialmente mortales o crónicas. En los niños los síntomas pueden ser sutiles o ausentes y se pueden desarrollar con el tiempo ${ }^{(4)}$. Entre las manifestaciones clínicas más frecuentes se describen: tos, disnea, dolor torácico, dolor pleural, hemoptisis ${ }^{(1)}$. Tomando en cuenta estas consideraciones, cabe destacar que todo niño que curse con alguna 


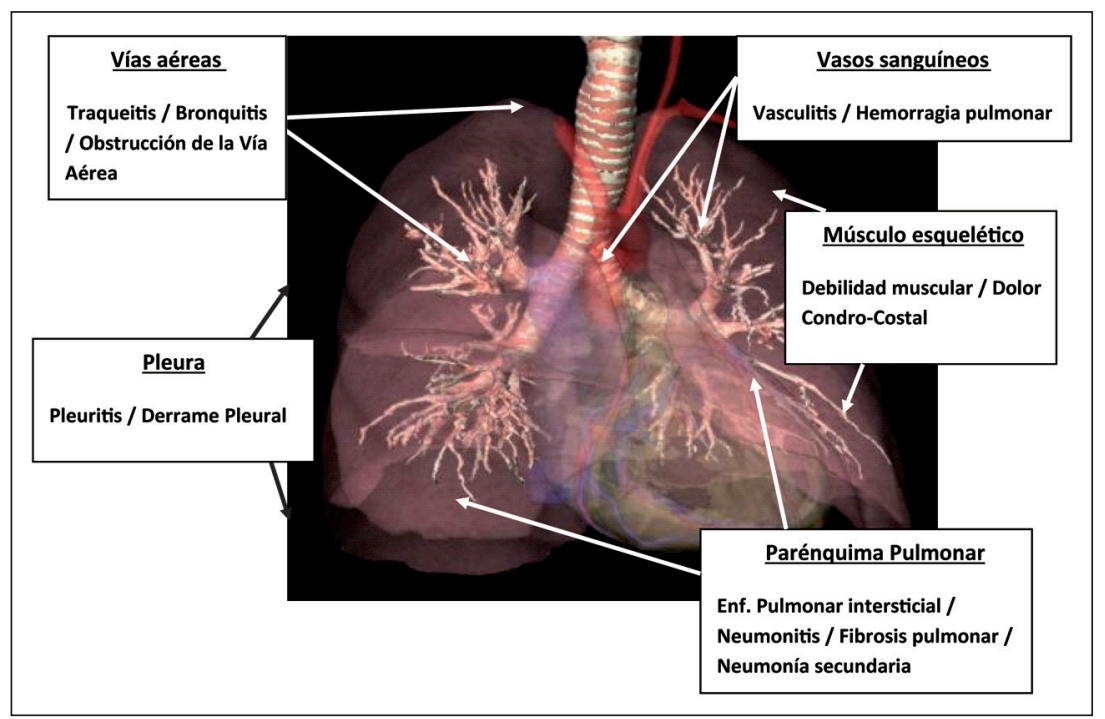

Figura I. Alteraciones respiratorias producidas por las enfermedades autoinmunes. Modelo Anatómico tomado con permiso de: http:// www.msdcontrol.com/services/emed/common/ rotatable/systems/Respiratory/Resp_LI F29.jpg de estas patologías tiene un riesgo elevado de afectación respiratoria, por lo tanto, debe ser evaluado integralmente y en forma multidisciplinaria.

Las enfermedades reumáticas, también llamadas colagenopatías, abarcan un amplio grupo de entidades. Aunque la experiencia en los pacientes pediátricos es escasa y limitada, en la actualidad recientes publicaciones nos brindan una información más clara al respecto, de gran ayuda en nuestra práctica diaria. A continuación se presenta una revisión de la literatura de las principales patologías reumáticas que afectan a los niños y su impacto en la vía respiratoria.

\section{LUPUS ERITEMATOSO SISTÉMICO}

Lupus Eritematoso Sistémico (LES) es una enfermedad autoinmune con base genética, su inicio en niños y adolescente ocurre en un $20 \%$ de los casos y de estos las manifestaciones respiratorias en niños se presentan en un 20 a $30 \%$ de los pacientes, el más frecuente es el compromiso pleural, aunque también se han descrito neumonitis aguda, enfermedad pulmonar intersticial (EPI), hemorragia alveolar, disfunción diafragmática e hipertensión pulmonar ${ }^{(5)}$ (Tabla I). En la mayoría de los casos con inicio en la edad pediátrica, presentan alteraciones en las pruebas de función pulmonar y hallazgos en la tomografía de alta resolución sin haber desarrollado síntomas respiratorios. En la pleuritis con o sin derrame, el paciente puede presentar dolor pleural y frote a la auscultación, la toracocentesis, en caso de derrame suele ser diagnóstica y terapéutica. La aparición súbita de patrones alveolares difusos, disnea, desaturación y descenso en el hematocrito hace sospechar la presencia de hemorragia alveolar. La enfermedad intersticial crónica es más difícil de diagnosticar porque es de instalación progresiva, con evidencia de patrones micronodulares, engrosamientos septales, presentes en tomografía de alta resolución (HRTC) y no en Rx. Simples (Figura 2). La bronquiolitis obliterante es una complicación rara pero severa, se confunde con procesos neumónicos agregados a paciente con LES, su diagnóstico la mayoría de las veces es

\section{Tabla I. Manifestaciones pulmonares en niños con LES}

\begin{tabular}{|c|}
\hline Manifestaciones pulmonares en niños con LES \\
\hline Pleuritis \\
\hline Derrame pleural \\
Neumonitis aguda \\
\hline Enfermedad pulmonar intersticial \\
\hline Hemorragia alveolar \\
\hline Fibrosis pulmonar \\
\hline Disfunción diafragmática \\
Neumonías con o sin derrame pleural \\
Bronquiolitis obliterante \\
Hipertensión pulmonar \\
\hline
\end{tabular}

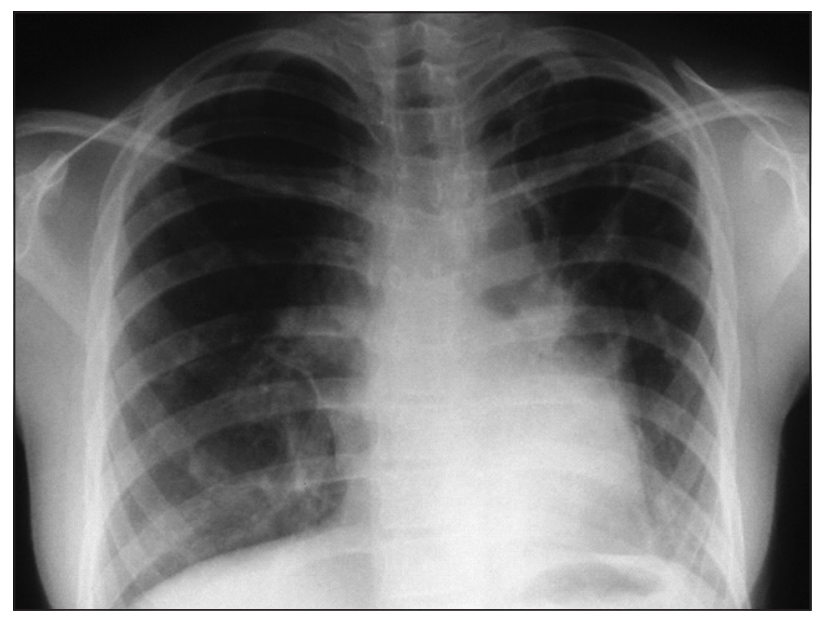

Figura 2. Adolescente femenina de 12 años con LES presenta lesión intersticial con patrón reticular predominante. Imagen propiedad de los autores. 
por biopsia. La hipertensión pulmonar suele presentarse en estados tardíos de la enfermedad y muchas veces asociada a enfermedad intersticial y también se puede observar "el síndrome de reducción pulmonar" con elevación diafragmática y reducción del volumen pulmonar secundario a fibrosis intersticial, pleural y disfunción diafragmática produciendo atrapamiento pulmonar ${ }^{(5,6)}$ (Tabla I).

Bestford y colaboradores en el 2005 reportaron en el Reino Unido una serie de casos de 29 niños diagnosticados entre 1995 al 2003, presentando manifestaciones respiratorias 8 pacientes, 4 EPI, 2 compromiso pleural, I hemorragia alveolar y I neumonitis lúpica, 6 pacientes presentaron primero manifestaciones respiratorias antes de hacerse el diagnóstico de LES(7). En Noruega Libelly y col, en 2006 presentaron un estudio de cohorte de 60 pacientes con LES de inicio en la infancia, I I ( $18 \%)$ presentaban disnea al ejercicio, 5 (8\%) tos crónica, 19 (31\%) pleuritis, 8 pacientes presentaron micronódulos en la tomografía de alta resolución $(\text { HRTC) })^{(8)}$.

En la Rx simple los hallazgos clásicos son: derrames pleurales, patrones intersticiales en Vidrio despulido perihiliares y patrones alveolares de consolidación neumónicas. En cambio en la HRTC se pueden documentar patrones reticulares y micronodulares más tempranamente. Según algunas series las pruebas de función pulmonar en estos pacientes presentan alteraciones de un 10\% hasta un 70\%, con predomino de patrón obstructivo bronquial en los estados iniciales de la enfermedad y restrictivos en afectación intersticial avanzada. Las alteraciones en la difusión de monóxido de carbono (DLCO) están presentes aproximadamente entre el 20 al 50\% de los casos, siendo esta un hallazgo precoz antes de presentar síntomas respiratorios o hallazgos tomográficos ${ }^{(7,9)}$.

\section{ARTRITIS JUVENIL IDEOPÁTICA (AJI)}

Los términos Artritis Reumatoide Juvenil (ARJ) utilizado por el Colegio Americano de Reumatología y Artritis Crónica Juvenil (ACJ) utilizado por la Liga Europea contra el Reumatismo han decidido modificarse por lo que se adopta de forma mayoritaria la denominación de Artritis idiopática Juvenil (AlJ) propuesto por la Liga Internacional contra el Reumatismo como un término intermedio, para indicar que es un proceso de la infancia (menores de 16 años) que se caracteriza principalmente por artritis que persiste durante 6 sem como mínimo y que no se puede atribuir a ninguna causa conocida actualmente. Este término idiopática evita de una forma rotunda el crónico y el reumatoideo y reconoce que el origen de estas enfermedades es todavía desconocido, por tal motivo se le denomina artritis idiopática juvenil $(\mathrm{All})()^{(10)}$.

Se definen 8 subtipos de All:

I. Poliarticular con factor reumatoideo (FR) negativo.

2. Poliarticular con FR positivo.

3. Oligoarticular: menos de 5 articulaciones involucradas.

4. Oligoarticular extendido: más de 5 articulaciones involucradas.

5. Sistémica.

6. Psoriásica.

7. Artritis-entesitis.

8. Otras (II).
La All sistémica seguida de la All Poliarticular FR+, son los subtipos más comúnmente asociado a compromiso pulmonar. Estas afecciones pulmonares son poco frecuentes pero puede tener consecuencias perjudiciales cuando no es reconocida y tratada. En los niños con All de inicio sistémico la pleuritis es la causa más frecuente de afectación pleuropulmonar pero rara vez se observa en poliarticular All(I). La característica de un derrame exudativo reumatoide es una leucocitosis linfocítica, baja glucosa, elevación de LDH y proteínas, disminución del complemento(II). Hay pocos reportes de pacientes con Hipertensión Pulmonar primaria, fibrosis intersticial difusa asociada con la presencia de granulomas intraalveolares e intersticiales, neumomediastino y Hemosiderosis pulmonar ${ }^{(12)}$. En cuanto a las pruebas de función pulmonar se observa patrón restrictivo y disminución del DLCO(II). El lavado broncoalveolar permite el análisis de células inflamatorias y descarte de patologías infecciosas como en otras entidades ya descritas(II). La biopsia pulmonar es hasta ahora estándar de oro para la evaluación de la enfermedad pleuropulmonar en la AlJ. En una gran mayoría de niños con All y biopsia son positivos, entre los hallazgos histológicos se encuentra la neumonitis intersticial linfoide entre otras $\left({ }^{(I)}\right.$. En relación al tratamiento los corticosteroides han sido el pilar de la terapia. Es importante destacar que la experiencia en el tratamiento de niños con afección pleuropulmonar y JIA es limitada y ha producido resultados variables desde resolución completa de la enfermedad hasta evolución a la cronicidad. Existen agentes inmunosupresores utilizados con resultados variables ${ }^{(I)}$.

\section{ESCLEROSIS SISTÉMICA JUVENIL}

Es una entidad poco frecuente, definida en adultos como esclerodermia o esclerosis sistémica y definida desde 2007 como Esclerosis Sistémica Juvenil (ESJ), son los pacientes que presenta menos de 16 años y deben tener presente el criterio principal el cual es esclerosis o induración cutánea proximal y al menos 2 de 20 criterios secundarios, agrupados en nueve categorías principales ${ }^{(I)}$, entre los criterios respiratorios están fibrosis pulmonar documentada por HRTC, DLCO disminuida, HAP(13). Representa el $5 \%$ de todos los casos de Esclerodermia, con un inicio promedio a los 8 años, y es hasta 4 veces más frecuente en niñas. Los síntomas más frecuentes son la induración cutánea en $82 \%$ de los casos, seguido de la afección respiratoria 42\%. En general la presentación es menos severa que en los adultos, teniendo menos lesiones en piel y menor grado de afectación en órganos. Hay un grupo de pacientes que entran en el síndrome de solapamiento, presentando signos de dermatomiositis y polimiositis ${ }^{(14)}$.

En un estudio retrospectivo en Italia, Panigada y col, reportaron 17 pacientes con ESJ, evaluándolos con pruebas de función respiratoria (PFR), HRTC y revisando su evolución en el tiempo, el 58,8\% de los pacientes presentó una diminución en VEF, CVF y DLCO, siendo progresiva la disminución de VEF, y CVF, los hallazgos en la HRTC fueron evaluados por un sistema de puntos, a mayor lesiones pulmonares mayor puntaje, desde engrosamiento fibrótico de tabiques pulmonares, micronódulos, patrón de vidrio despulidos, 
hasta lesiones en panal de abeja y alteración de la arquitectura pulmonar. Se demostró una relación inversa entre $\mathrm{VEF}_{\text {, l }}$ la CVF y las lesiones encontrada en la HRTC, sobre todo en la pérdida de la CVF, sugiriendo que el seguimiento respiratorio de estos pacientes puede hacerse con PFR, disminuyendo la necesidad de exposición a radiaciones frecuentes ${ }^{(15)}$.

\section{DERMATOMIOSITIS JUVENIL}

La Dermatomiositis juvenil es una enfermedad autoinmune caracterizada clásicamente por Rash cutáneo, debilidad muscular proximal y elevación de las enzimas musculares. El daño primario es una vasculitis de pequeños vasos con marcador Jo-I positivo. Los hallazgos típicos respiratorios en los adultos llegan a ser 30 a 50\% dada por enfermedad pulmonar intersticial (ILD) (neumonitis intersticial usual) son menos frecuentes en la infancia ${ }^{(I)}$, aunque raras, serias manifestaciones en niños han sido descritas como bronquiolitis obliterante, distrés respiratorio agudo, falla respiratoria por afección severa de las musculatura respiratoria.

Un estudio de casos y controles con 59 pacientes con Dermatomiositis Juvenil y una edad media de 16,8 años, fueron comparados en su función pulmonar con controles, la capacidad pulmonar total y la difusión de $\mathrm{CO}$ disminuida en forma significativa, en $26 \%$ y $49 \%$ de los pacientes respectivamente. En HRTC se encontró alterada en $37 \%$ de los pacientes, ILD |4\%, enfermedad de la vía aérea |4\%, calcinosis de la pared torácica I4\%(16). Al igual que otras enfermedades autoinmunes en la infancia suelen los pacientes no tener síntomas respiratorios relevantes pero con alteraciones funcionales y anatómicas subclínicas presentes. La terapéutica principal se basa en corticosteroides y metrotexate, cobrando cada vez más relevancia las terapias inmunológicas. Los pulsos de metil prednisolona pueden estar indicados en casos severos con compromiso de la musculatura respiratoria.

\section{SÍNDROME DE SJÖGREN (SS)}

Es una enfermedad crónica autoinmune que afecta principalmente las glándulas lagrimal y salival con grado variable de implicación sistémica; puede ocurrir sólo (SS primario) o asociado a otras enfermedades autoinmunes como artritis reumatoidea, lupus eritematosos sistémico o esclerodermia (SS secundario) $)^{(17)}$.

Es extraordinariamente raro en pediatría con una edad media de inicio de 10 años. Dentro de los exámenes realizados para el diagnóstico los autoanticuerpos encontrados positivos son ANA, La (SS-B), factor reumatoideo y Ro (SS$A)^{(11)}$, este último asociado frecuentemente con presencia de manifestación extraglandular sobre todo la enfermedad pulmonar intersticial(12): la neumonía intersticial linfocítica y no específica, más comúnmente encontrada en SS secundario(18). Es importante resaltar que uno de los síntomas respiratorios es tos seca confundiéndonos con alergia respiratoria, asma o bronquitis por largo tiempo(12).

No hay consenso para el manejo en niños. Los corticoesteroides parecen ser efectivos en la prevención de la progresión y a veces causar la regresión de la enfermedad. Estudios realizados en pequeños grupos de pacientes tratados con azatioprina con y sin esteroides han tenido mejora de la enfermedad pulmonar que el grupo placebo, sugiriendo que la azatioprina puede ser efectivo en el tratamiento de SS ${ }^{(18)}$.

\section{ENFERMEDAD MIXTA DEL COLAGENO}

Es considerado como un síndrome de superposición, sus manifestaciones son una mezcla de características de LES, artritis, dermatomiositis y escleroderma. La enfermedad pulmonar intersticial, la pleuritis y la hipertensión arterial son las formas más comunes de afectación pulmonar. Estos pacientes pueden cursar con dismotilidad esofágica que es causa de broncoaspiración y mayor riesgo de neumonías por aspiración. En cuanto a la función pulmonar al igual que otras enfermedades reumatológicas los pacientes pueden tener alteración de tipo restrictivo y ser asintomáticos, así como disminución del DLCO. En cuanto a la tomografía se evidencia engrosamiento septal, opacidad en vidrio esmerilado y en etapas más tardías e imágenes en panal de abejas. A los pacientes con clínica y alteración de la función pulmonar se les debe indicar tomografía del tórax y de acuerdo a los hallazgos si no demuestran nada concluyente se les debe realizar lavado broncoalveolar. En cuanto al tratamiento este debe ser con inmunosupresores y esteroides por período por lo menos de 3 a 6 meses $(11)$.

\section{VASCULITTIS}

El término vasculitis denomina a un grupo de enfermedades clínicamente heterogéneas, pero con un sustrato histológico común: la presencia de un infiltrado inflamatorio en el espesor de la pared de los vasos. Es la característica predominante de un gran número de enfermedades y síndromes infantiles ${ }^{(19)}$.

Las vasculitis en pediatría se clasifican en primaria de pequeño, mediano, grandes vasos y ANCA positivas y secundarias entre estas relacionadas a infección, enfermedades del tejido conectivo, hipersensibilidad a fármacos, malignidad, post trasplante de órganos entre otras ${ }^{(20)}$.

La afectación pulmonar de la vasculitis puede desarrollarse en cualquier vaso, independientemente de su tamaño. La afectación de los pequeños vasos (microvasculatura) se conoce como capilaritis pulmonar, este es un diagnóstico patológico, pero su presencia hace necesario descartar cualquier tipo de enfermedad subyacente como las enfermedades autoinmunes sistémicas o vasculitis asociadas a $\mathrm{ANCA}^{(21)}$.

La consecuencia más grave de la capilaritis pulmonar es la hemorragia alveolar, la cual se caracteriza por infiltrados alveolares difusos, de aparición brusca hemoptisis y caída del hematocrito y/o hemoglobina (Figura 3). Es muy característico y orientador en el diagnóstico la presencia de hemosiderófagos en el lavado broncoalveolar, ya que los macrófagos alveolares fagocitan a los eritrocitos que fluyen de la lesión en la membrana basal del endotelio vascular hasta el alveolo(2).

Es un cuadro potencialmente mortal y con evolución 


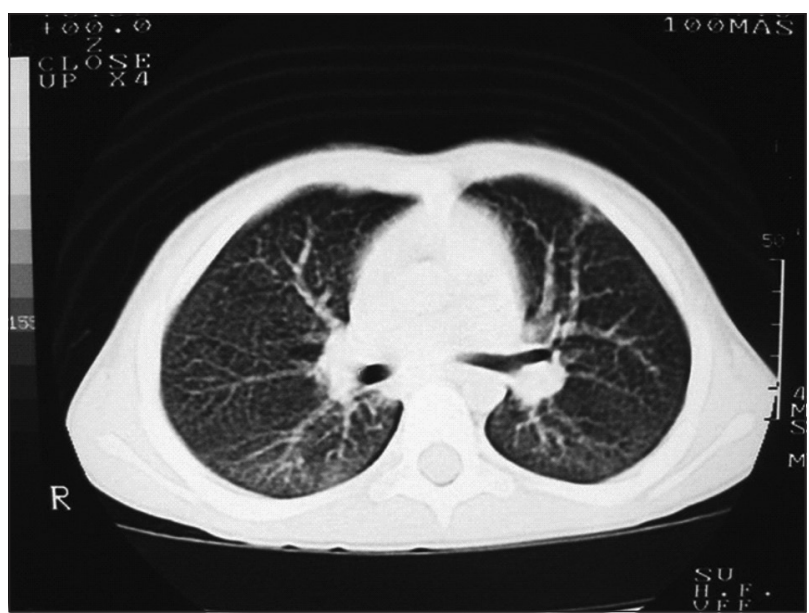

Figura 3. Prescolar de 6 años con hemosiderosis pulmonar secundaria a vasculitis, lesiones alveolo-intersticiales difusas. Imagen propiedad de los autores.

clínica impredecible. En cuanto a los estudios diagnósticos en la radiografía del tórax es inespecífica, muestra infiltrados alveolares localizados o difusos, que pueden ser mejor evidenciados y confirmados en tomografía de tórax de alta resolución. Desde el punto de vista funcional se evidencia una disminución de la capacidad de difusión del monóxido del carbono (DLCO). Si la condición del paciente lo permite es mandatorio realizar lavado broncoalveolar ya que ayuda a establecer el diagnóstico y descartar otras entidades. La presencia de 20\% de hemosiderófagos es diagnóstico de hemorragia pulmonar ${ }^{(21)}$. El manejo terapéutico dependerá de la gravedad así como de las manifestaciones extrapulmonares. Los casos de gravedad deben ser manejados en una unidad de cuidados respiratorios con soporte respiratorio(21).

En cuanto al tratamiento es poca la experiencia en pediatría por ser entidades pocos frecuentes. Se describen en adultos un tratamiento de inducción con inmunosupresores en casos de que el paciente presente estabilidad y no precise soporte ventilatorio con el objetivo de controlar la actividad de la vasculitis y alcanzar un estado de remisión, seguida de una fase de mantenimiento para prevenir las recidivas ${ }^{(21)}$.

Al revisar la literatura encontramos el término vasculitis pulmonares para definir un grupo de enfermedades en las que se produce inflamación y destrucción de los vasos y el parénquima pulmonar ${ }^{(22)}$. Se clasifican en las que afectan constantemente al pulmón (enfermedad de Churg Strauss, enfermedad de Weneger, Poliangeitis microscópica) y la de afectación pulmonar no constante (Púrpura de SchonleinHenoch, arteritis de Takayasu, arteritis de células gigantes, criglobulinemia, poliarteritis nodosa, enfermedad de Beçhet, etc. $)^{(21,22) .}$

La purpura de Schonlein-Henoch y la granulomatosis de Wegener son los dos cuadros clínicos descritos en niños, este últimos como presentación clínica excepcional ${ }^{(23)}$. La Granulomatosis de Wegener se puede presentar a cualquier edad pero principalmente en los adultos. Afecta constantemente la vía respiratoria con alteración inicial de las vía respiratoria alta en forma de rinorrea, ulceración, sinusitis y destrucción del tabique nasal. El pulmón se ve afectado por la presencia de nódulos pulmonares, cavidades y posible hemorragia pulmonar, así como alteración de la función respiratoria con un patrón obstructivo, mixto y disminución del DLCO. Cursa además con otras manifestaciones generales como glomerulonefritis, alteraciones cutáneas, artralgia, síntomas de hemorragia digestiva. Para orientar el diagnostico es importante la determinación serológica de ANCA. El tratamiento se basa en el uso inicial de ciclofosfamida y esteroides. Se ha preconizado como tratamiento complementario cotrimoxazol $^{(23)}$. Aunque la purpura de Schonlein-Henoch es la vasculitis más frecuente en la infancia la afectación pulmonar es rara, se han descrito episodios de hemorragia pulmonar y lesión intersticial, además de disminución del DLCO.

\section{MANIFESTACIONES RESPIRATORIAS SECUNDARIA AL TRATAMIENTO DE LAS ENFERMEDADES REUMÁTICAS}

El tratamiento de las enfermedades reumáticas puede tener efectos secundarios respiratorios, pero no son fáciles de identificar, deben descartarse primero las repercusiones de la enfermedad de base en el sistema respiratorio, los procesos secundarios agregados como neumonías, tromboembolismos, aspiraciones crónicas, etc. Muchos pacientes se encuentran en tratamiento con múltiples drogas que pueden tener efectos secundarios respiratorios. El diagnóstico muchas veces es por exclusión. Los síntomas pueden ser inespecíficos como disnea, tos seca o dolor pleural, fiebre, presencia de crepitantes. Las drogas relacionadas son: colchicina, ciclofosfamida, D-penicilinasa, sales de oro, metrotexate, AINES(5). Los nuevos agentes biológicos como el Anti TNF pueden producir enfermedad pulmonar intersticial de mal pronósti$\mathrm{CO}^{(24)}$. Destacando que en el tratamiento Metrotexate puede presentar hasta un 3\% de neumonitis intersticial(25). Los efectos secundarios al tratamiento con corticosteroides son bien conocidos, con repercusión sistémica múltiple y pocos pulmonares. Se describen cinco síndromes respiratorios secundarios a medicamentos (Tabla 2).

Los mecanismos de acción pueden ser por reacciones de hipersensibilidad, citotoxicidad directa, respuesta linfocítica anormal, incremento de la permeabilidad capilar y alteraciones en la función plaquetaria. El tratamiento es con medidas

\section{Tabla 2. Síndromes respiratorios asociados a medicamentos}

\section{Síndromes respiratorios asociados a medicamentos}

- Neumonitis por hipersensibilidad (AINES, metrotexate, penicilamina, sales de oro)

- Alveolitis crónica y fibrosis (AINES, metrotexate, penicilamina, sales de oro)

- Sd riñón-pulmón, similar al Sd. Goodpasture (penicilamina)

- Bronquiolitis obliterante (sales de oro, penicilamina)

- Edema pulmonar no cardiogénico (AINES, salicilatos, colchicina) 
de sostén, utilización de esteroides sistémicos, hemoderivados en caso de trombocitopenia o anemia importante y el cambio en tratamiento o modificación en la dosificación.

\section{CONCLUSIONES}

Las consideraciones anteriores nos indican, que en todo niño con riesgo elevado se debe tener una alta sospecha diagnóstica de probable afectación pulmonar y ser evaluado integralmente. En el plan de estudio de estos pacientes es importante realizar:

a) Radiología del tórax Pa y lateral.

b) Pruebas de función pulmonar: entre las principales pruebas a realizar están la espirometría, gases arteriales y DLCO. Es poca la experiencia en pacientes pediátricos con enfermedad reumatológica; sin embargo, una investigación reciente realizados en niños han demostrado alteración de la función pulmonar en estos pacientes aun sin síntomas respiratorios. De allí parte la recomendación de este estudio de realizar pruebas de función pulmonar a todos los pacientes con enfermedades reumáticas al menos una vez al año incluso en ausencia de síntomas respiratorios con el fin de detectar alteración temprana, optimizar tratamiento y evitar complicaciones relacionadas $^{(5)}$.

c) Tomografía del tórax de alta resolución: es la técnica de imagen de elección para detectar, caracterizar y determinar el grado y la extensión de afectación pulmonar en paciente con sospecha clínica de enfermedades sistémicas, que en las primeras etapas puede no ser visible en las radiografías de tórax ${ }^{(6)}$. La CT de alta resolución puede evidenciar lesiones pulmonares en niños con muy pocos o ningún síntoma respiratorio y pruebas de función pulmonar normal(6). En una reciente investigación realizada en pacientes pediátricos se describen los principales patrones encontrados en enfermedades del tejido conectivo siendo las frecuentes el patrón en vidrio esmerilado, engrosamiento septal, panal de abejas, fusión pleural y pericárdica. Estos patrones varían en frecuencia según el tipo de enfermedad del tejido conectivo(6).

d) Baciloscopía, gran y cultivo de esputo a todo niño con tos y expectoración.

e) Estudio del líquido pleural debe ser completo, con evaluación desde el punto de vista citológico, bacteriológico e inmunológico.

f Lavado bronco alveolar, permite evaluar marcadores de inflamación, celuraridad, hemosiderófagos, además de ser un procedimiento terapéutico en algunos casos.

g) Biopsia pulmonar de acuerdo a la severidad del caso y de dudas diagnósticas.

\section{REFERENCIAS}

I. Espada G, Linares C. Manifestaciones Respiratorias de Colagenopatía. En: Macri C, Teper A. Enfermedades Respiratorias Pediátricas. Mc Graw Hill, 2003, pp 513-22.

2. Dell Beng $\mathrm{S}$, Schneider R. Pulmonary involvement in the systemic inflammatory diseases of childhood. In: Kendig and Chernick's disorders of the respiratory tract in children. Sauders Elservier, 2012, pp 822-32.

3. Eid NS, Buchino JJ, Schikler. Pulmonary manifestation of rheumatic diseases. Pediatr pulmonol Supp 1999; 18: 91-2.

4. Ravinovich CE. Pulmonary complications of childhood rheumatic disease. Paediatr Respir Rev 2012; 13: 29-36.

5. Platzker AA. Pulmonary involvement in rheumatoid disorders of childhood. In: Chernick V, Boat T, Wilmontt R, Bush A. Kendig's Disorders of the respiratory tract in children. Saunders Elsevier, 2006, pp 949-77.

6. García Peña P, Boixadera H, Barber I, Toran N, Lucaya J, et al. Thoracic Findings of Systemic Diseases at High-Resolution CT in Children. Radiographics 201 I; 31: 465-82.

7. Beresford MW, Cleary AG, Sills JA, Couriel J, Davidson JE. Cardiopulmonary involvement in juvenile systemic lupus erythematosus. Lupus 2005: 14; 152-8.

8. Libelly B, Aaløkken TM, Johansen B, Førre $\varnothing$. Pulmonary involvement in patients with child-onset systemic lupus erithemayosus. Clin Exp Rheumatol 2006; 24: 203-8.

9. Balachandar D, Nandalike K, Matta Arroyo E, Blumer S, Goilav B, et al. Pulmonary Manifestations In Children With Systemic Lupus Erythematosus. Am J Respir Crit Care Med 20 12; I85: A6 126.

10. González Pascual E. Nueva denominación y nueva clasificación de la Artritis crónica juvenil. Por qué? Avances en Especialidades Pediátricas. Sevilla 2000. www.sepeap.org/imagenes/secciones/ Image/USER/AEP 20/I I/2012.

II. Ravinovich CE. Pulmonary complications of Childhood Rheumatic Disease. Paediatr Respir Rev 2012; 13: 29-36.

12. Domingues V, Felix Rodrigues M, Costa Diniz C, Gasparello de Almeida R, Sztajnbox F. The Respiratory tract and juvenile rheumatic diseases. Rev Bras Reumatol 2011; 51: 88-96.

13. Zulian F, Woo P, Athreya BH, Laxer RM, Medsger TA Jr, et al. The Pediatric Rheumatology European Society/American College of Rheumatology/European League against Rheumatism provisional classification criteria for juvenile systemic sclerosis. Arthritis Rheum 2007; 57: 203-12.

14. Zulian F. Esclerosis sistémica y esclerodermia localizada en la infancia. Rheum Dis Clin N Am 2009; 34: 239-55.

15. Panigada S, Ravelli A, Silvestri M, Granatt C, Magni-Mazoni S, et al. HRCT and Pulmonary Function Tests in Monitoring of Lung Involvement in Juvenile Systemic Sclerosis. Pediatr Pulmonol 2009; 44: 1226-34.

16. Sanner H, Aaløken TM, Gran JT, Sjaastad I, Johansen B, et al. Pulmonary outcome in juvenile dermatomyositis: a case-control study. Ann Rheum Dis 2011; 70: 86-91.

17. Civilibal M, Canpolat N, Yurt A, Kurugoglu S, Erdamar S, et al. A Child with Primary Sjogrën Syndrome and review of the Literature. Clin Pediatr (Phila) 2007; 46: 738-42.

18. Vece TJ, Fan LL. Interstitial Lung Disease in Children older Than 2 Years. Pediatr Allerg Inmunol Pulmonol 20 I2; 23: 33-4I.

19. Calvo Penandés I. Clasificación de las vasculitis en el niño. An Pediatr (Barc) 2005; 62: 263-6.

20. Dedeoglu F, Sundel RP. Vasculitis in children. Pediatr Clin North Am 2005; 52: 547-75.

21. Martín Suñé N, Ríos Blanco J. Afectación pulmonar de la vasculitis. Arch Bronconeumol 2012; 48: 410-8.

22. Falces A, Maza C. Manifestaciones pulmonares de las enfermedades del tejido conectivo. Vasculitis pulmonares. 
Eosinofilias pulmonares. www.neumosur.net/files/EB04-36\%20 conectivopatias.pdf /6/1 ///2.

23. Martínez Gómez M, Puerta Vilchez M. Martínez Vernal J, Martínez Cañavate A. Vasculitis y síndromes hemorrágicos pulmonares. An Esp Pediatr 2002; 56(supl 2): 44-5.

24. Pérez Álvarez R, Pérez de Lis M, Díaz Lagares C, Pego Reigosa J,
Retamozo S, et al. Interstitial lung disease induced or exacerbated by TNF-targeted therapies: analysis of 122 cases. Semin Arthritis Rheum 201 I; 41: 256-64.

25. Trinidad Guerrero D, Ladin L, Hamdan P, Bermúdez P, García T, et al. Enfermedad Intersticial pulmonar asociada a Metrotexate. Neumol Cir Tórax 2009; 68: 35-40. 